\title{
IncRNA MALAT1 Mediates Osteogenic \\ Differentiation and Apoptosis in Osteoporosis by Regulating the miR-485-5p/WNT7B Axis
}

\section{Yuan Zhou}

the First Hospital of Jilin University

\section{Zhuo Xu}

China-Japan Union Hospital of Jilin University

\section{Yuanyi Wang}

the First Hospital of Jilin University

\section{Qiang Song}

China-Japan Union Hospital of Jilin University

\section{Ruofeng Yin ( $\nabla$ yrf_wind@jlu.edu.cn )}

Department of Clinical Laboratory, the First Hospital of Jilin University https://orcid.org/0000-00021083-4928

\section{Research}

Keywords: osteoporosis, MALAT1, osteogenic differentiation, WNT7B

Posted Date: October 5th, 2021

DOI: https://doi.org/10.21203/rs.3.rs-885726/v1

License: (c) (i) This work is licensed under a Creative Commons Attribution 4.0 International License. Read Full License 


\section{Abstract}

Background: Accumulating evidence demonstrates that long non-coding RNAs (IncRNAs) are associated with the development of osteoporosis. This study aimed to investigate the effects of metastasisassociated lung adenocarcinoma transcript 1 (MALAT1) on osteogenic differentiation and cell apoptosis in osteoporosis.

Methods: hindlimb unloading (HU) was performed to establish osteoporosis model in vivo. MicroCT was applied for pathological analysis. Microgravity (MG) was used to construct osteoporosis in vitro. The mRNA and miRNA expression was determined using RT-qPCR. Protein expression was determined using western blot. The binding sites between miR-485-5p and MALAT1/Wnt family member 7B (WNT7B) was predicted by bioinformatics analysis and verified by luciferase and RNA pull-down assays. Cellular functions were determined by ALP staining, Alizarin red staining, and flow cytometry assays.

Results: MALAT1 expression was downregulated in HU mice and MG treated MC3T3-E1 cells. However, overexpression of MALAT1 upregulated the expression of Bmp4, Col1a1, Spp1, and enhanced ALP activity. Additionally, overexpression of MALAT1 inhibited apoptosis, decreased Bax and caspase-3 levels, and increased Bcl-2 level. Moreover, MALAT1 overexpression improved bone phenotype in vivo. MALAT1 functioned as a ceRNA to upregulate WNT7B. Overexpression of miR-485-5p rescued the promotion of osteogenic differentiation and the inhibition of apoptosis induced by MALAT1. Knockdown of WNT7B abolished the facilitation of osteogenic differentiation and the suppression of apoptosis induced by downregulation of miR-485-5p.

Conclusion: In conclusion, MALAT1 promoted osteogenic differentiation and inhibited cell apoptosis through miR-485-5p/WNT7B axis, which suggested that MALAT1 is a potential target to alleviate osteoporosis.

\section{Background}

Osteoporosis is a common systemic bone disease, which is characterized by bone loss and bone microstructure disorders $(1,2)$. ,The incidence of osteoporosis with aging and and reach as high as $13.2 \%$, which seriously affects the health and quality of life of the elderly (3). High glucose, anorexia nervosa, aging and osteoblast dysfunction are the direct causes of osteoporosis (4). In normal bone tissue, osteoblasts and osteoclasts maintain a dynamic balance; however, this imbalance may lead to various bone diseases, including osteoporosis (5). Osteoblasts play a key role in bone formation or bone repair $(6,7)$. Therefore, to promote osteogenic differentiation of osteoblasts may help alleviate osteoporosis.

LncRNAs are a group of non-coding RNAs longer than $200 \mathrm{nt}$ which were considered as by-products produced by RNA polymerase II during transcription $(8,9)$. However, numerous researches have confirmed that IncRNAs exert multiple functions, such as direct or indirect transcriptional regulation, transcription factor isolation, and protein or RNA regulation (10). Metastasis-associated lung adenocarcinoma 
transcript 1 (MALAT1) located at human chromosome 11q13 and mouse chromosome 19q with a length of $8.5 \mathrm{~kb}$ (11), and is a well-studied IncRNA in human diseases including osteoporosis (12). A previous report has revealed that MALAT1 enhances osteoblast activity to alleviate osteoporosis. Besides, MALAT1 promotes osteoporosis progression through inhibiting osteogenic differentiation of bone marrow mesenchymal stem cells (13). However, the roles of MALAT1 in preosteoblast cells were still largely unknown.

Wnt family member 7B (WNT7B) is a member of WNT family, which is involved in various biological processes, such as proliferation, apoptosis, inflammatory response, and metabolisms (14). WNT signaling is classified into canonical and noncanonical WNT pathway (15). The canonical Wnt pathway is highly evolutionary conserved and affects $\beta$-catenin. Previous studies evidence that WNT7B initiates $\beta$ catenin to regulate the occurence and development of bone disorders, including $(16,17)$. For instance, Chen et al. (18) reveal that WNT7B WNT7B rescues glucocorticoid-induced bone loss and suppresses secondary cause for osteoporosis. Moreover, WNT7B regulates glucose metabolism and promotes bone formation (19). Herein, hindlimb unloading (HU) and microgravity (MG) assays were performed to establish osteoporosis model in vivo and in vitro. We found that MALAT1 functioned as a ceRNA to upregulate the expression of WNT7B via sponging miR-485-5p. These findings extended our

understanding of the function and mechanism of MALAT1 in osteoporosis development and may provide new target for the treatment of osteoporosis.

\section{Materials And Methods}

\section{Establish osteoporosis mice model}

Male C57BL/6J mice ( 6 months old) were provided by VitalRiver Laboratory Animal Co.Ltd (Beijing, China). These animals were maintained under the condition of $21^{\circ} \mathrm{C}$, with $12 \mathrm{~h}$ light and $12 \mathrm{~h}$ dark cycle. To establish unloading model, the mice were suspended by the tail at an angle of approximately $30^{\circ}$ with their forelimbs touching the floor which allowed them to move and access food and water for free. The tail suspension lasted for 3 weeks. Finally, these mice were sacrificed using overdose anesthesia (100 mg/kg pentobarbital). The bilateral femurs were dissected for Micro-computed tomography $(\mu \mathrm{CT})$ analysis. Animal studies were all approved by the Ethics Committee of China-Japan Union Hospital of Jilin University and were performed according to the approved guidelines. Then the bone tissues were collected and analyzed using Micro-CT scanner (Scanco Medical AG, Switzerland).

\section{Simulated microgravity treatment}

2D Rotating Wall Vessel Bioreactor (RWVB) clinostat was used to simulate microgravity. $1 \times 105$ of MC3T3-E1 cells were seededon cell climbing pieces. $1 \times 10^{5}$ MC3T3-E1 cells were seeded on coverslips. After culture for $24 \mathrm{~h}$, the climbing pieces were placed in a box $12.5 \mathrm{~mm}$ away from therotational axis. After the air bubbles were removed, the chambers were fixed in the clinostat and rotated around a 
horizontal axis at $28 \mathrm{rpm}$ for $15 \mathrm{~min}$. The vertical rotation groups were used as controls. The rotation process was taken at $37^{\circ} \mathrm{C}$ under $5 \% \mathrm{CO}_{2}$.

\section{Cell culture}

The mouse pre-osteoblast cell line MC3T3-E1 was purchase from American Type Culture Collection (ATCC, USA). Cells were cultured in a-MEM medium supplemented with $10 \%$ fetal bovine serum (FBS, Gibco), $100 \mathrm{U} / \mathrm{mL}$ penicillin, $100 \mu \mathrm{g} / \mathrm{mL}$ streptomycin and $110 \mathrm{mg} / \mathrm{mL}$ sodium pyruvate under $5 \%$ CO2 at $37^{\circ} \mathrm{C}$ in a humidified atmosphere. For osteogenic differentiation, a specific cultu was purchase from Cell bank of Chinese Academy of Sciences and cultured in DMEM (Gibco, USA) supplemented with 10\% fetal bovine serum (FBS, Gibco), $100 \mathrm{U} / \mathrm{mL}$ penicillin, $100 \mu \mathrm{g} / \mathrm{mL}$ streptomycin and $110 \mathrm{mg} / \mathrm{mL}$ sodium pyruvate under $5 \% \mathrm{CO}_{2}$ at $37^{\circ} \mathrm{C}$ in a humidified atmosphere. For osteogenic differentiation, a specific culture medium containing $100 \mathrm{nM}$ dexamethasone, $50 \mu \mathrm{M}$ ascorbic acid (Sigma), and $10 \mathrm{mM} \beta$ glycerophosphate was used.

\section{Cell transfection}

Empty vector (pcDNA3.1), MALAT1 (pcDNA3.1-MALAT1), siRNA (si)-nc, si-MALAT1, mimic negative control (nc), miR-485-5p mimic, inhibitor nc, miR-485-5p inhibitor, si-WNT7B were synthesized by Hanbio (Shanghai, China). MC3T3-E1 cells were seed in 6-well plates and transiently transfected using Lipofectamine $^{\mathrm{TM}} 2000$ (Invitrogen, Carlsbad, CA, USA) according to the instructions. After incubation of 6 $\mathrm{h}$, the medium was replaced by a complete medium.

\section{RT-qPCR}

Total RNA was isolated via TRIzol reagent (Invitrogen, Carlsbad, CA, USA). After the concentration determined the absorbance (A)260/A280 ratio, RNA was reverse transcribed to CDNA using SuperScript reverse transcriptases (for mRNA; Thermo Fisher Scientific, Waltham, MA, USA) and One Step PrimeScript miRNA cDNA Synthesis Kit (for miRNA; Takara, Tokyo, Japan). qPCR for mRNA was conducted by SYBR PCR Master Mix (GenePharma, Shanghai, China) with the conditions of $95^{\circ} \mathrm{C}$ for 3 min; 40 cycles of $95^{\circ} \mathrm{C}$ for $12 \mathrm{~s}$ and $62^{\circ} \mathrm{C}$ for $40 \mathrm{~s}$. For miRNA, qPCR was performed using SYBR Premix Ex Taq II (Perfect Real Time) (Takara, Tokyo, Japan) with the condition of $95^{\circ} \mathrm{C}$ for $10 \mathrm{~s}, 40$ cycles of $95^{\circ} \mathrm{C}$ for $5 \mathrm{~s}$ and $60^{\circ} \mathrm{C}$ for $20 \mathrm{~s}$. All samples were repeated three times. The expression level of genes was calculated using $2^{-\triangle \Delta C T}$ method. GAPDH and U6 were used as the internal control for mRNA and miRNA respectively.

\section{Luciferase reporter assay}

The luciferase reporter vector pGL3.1 carrying wild type binding cite (MALAT1-wt or WNT7B-wt) or mutant type binding cite (MALAT1-mut or WNT7B-mut) were constructed by RiboBio (Guangzhou, China). 293T cell was transfected with the MALAT1-wt or MALAT1-mut along with miR-485-5p mimic or mimic control. Renilla luciferase plasmid was transfected as a internal control. $48 \mathrm{~h}$ later, according to the instructions of the dual luciferase detection kit, a microplate reader was used to assess the firefly and Renilla luciferase activity in each group. The ratio of firefly fluorescence intensity of Renilla fluorescence intensity reflects the relative fluorescence intensity of each group. 


\section{RNA pull-down}

The biotinylated probe of miR-485-5p and the control probe were synthesized by Shenggong Biotech (Shanghai, China). Then the probe were incubated the probe with streptavidin-coated beads (Invitrogen, Carlsbad, CA, USA) at $25^{\circ} \mathrm{C}$ for $2 \mathrm{~h}$ to generate the probe-coated beads. The streptomycin beads were capable of binding to biotin. Cells were lysed to extract total RNA. After pretreatment of magnetic beads, RNA and beads were mixed. After separation, qPCR was used to quantify the relative expression of MALAT1 or WNT7B.

\section{Western blot}

The total proteins were extracted with lysis buffer containing protease inhibitor and phosphatase inhibitor cocktail (Sigma, CA, USA). $40 \mu \mathrm{g}$ proteins were separated in 10\% SDS-PAGE gel and then transferring onto a polyvinylidene fluoride (PVDF) membranes (Millipore, USA). Subsequently, the membranes were blocked in $5 \%$ non-fat dry milk in TBST buffer for $1 \mathrm{~h}$ at room temperature. Thereafter, the blots were incubated with the primary antibodies $4^{\circ} \mathrm{C}$ overnight followed by incubating with the horseradish peroxidase conjugated secondary antibody for another $2 \mathrm{~h}$ at room temperature. Finally, the blots were visualized using an enhanced chemiluminescence kit (Beyotime, Shanghai, China). The bands were quantified using ImageJ software.

\section{Alkaline phosphatase (ALP) activity}

The ALP activity of cells was evaluated using a Alkaline Phosphatase Assay Kit (Beyotime, Shanghai, China) according to the manufacture's instructios. Briefly, lysed cell were incubated with test buffer at $37^{\circ} \mathrm{C}$ for $10 \mathrm{~min} .100 \mu \mathrm{l}$ reaction stopping solution was used to terminate the reaction. A microreader (Biorad, USA) was used to assess the OD value at $490 \mathrm{~nm}$.

\section{ALP staining}

The ALP staining was conducted in the dark and assayed with the BCIP/NBT Alkaline Phosphatase Color Development according to the manufacture's instructions (Beyotime, Shanghai, China). The images were taken under a light microscope.

Alizarin red staining

After transfection, cells were collected and fixed with $4 \%$ paraformaldehyde for $15 \mathrm{~min}$. After washed with PBS thrice, cells were cultured with osteogenic induction medium for 3 weeks and then stained with alizarin red regents for $30 \mathrm{~min}$. Calcified nodules were captured using a microscope and calculated using spectrophotometric wavelength of $570 \mathrm{~nm}$.

\section{Flow cytometry}

After transfection and treatment, cells were washed with PBS thrice. Then the cells were stained with 2.5 $\mu \mathrm{g} / \mathrm{ML}$ of Annexin V-FITC and propidium iodide and incubate at room temperature in the dark for 15 
minutes. Finally, the cells were captured with a fluorescent microscope and evaluated by a FACSVerse flow cytometer system (BD, USA) with FlowJo software.

\section{Statistical analysis}

All the data are presented as means \pm standard deviation (SD). The statistical analyses were performed using SPSS version 17. Data were analyzed with one-way ANOVA and Student's test. $P<0.05$ was considered statistically significant. Pearson analysis was employed to evaluate the correlations between MALAT1 and miR-485-5p as well as between miR-485-5p and WNT7B.

\section{Results}

The ex pression of MALAT1 was decreased in MG-treated cells

As shown in Fig. 1A, MG exposure significantly deceased ALP expression. Moreover, MG treatment suppressed the mRNA and protein expression of Bglap, Runx2, and Col1a1 (Fig. 1B and C). Additionally, MG significantly decreased ALP activity and calcium deposit in MC3T3-E1 cells (Fig. 1D and E). IncRNAs play a vital role in the initiation and development of osteoporosis. Therefore, we determined the potentials of IncRNAs in MG-treated MC3T3-E1 cells. The results from cell sequencing showed that the expression of IncRNA MALAT1 was mostly downregulated. To further verify the roles of MALAT1 in osteoporosis, we determined its expression in MG-treated MC3T3-E1 cells. As shown in Fig. 1H, the expression of MALAT1 was significantly decreased in MG group.

\section{MALAT1 promoted osteogenic differentiation and inhibited cell apoptosis in MC3T3-E1 cells}

To explore the effects of MALAT1 on osteogenic differentiation, cells were transfected with MALAT1 overexpression plasmids. As shown in Fig. 2A, the expression of MALAT1 was significantly increased in MALAT1 group, suggesting cells were successfully transfected. Overexpression of MALAT1 significantly increased the expression of ALP, Bglap, Runx2, and Col1a1 (Fig. 2B-D). Moreover, Overexpressed MALAT1 significantly increased ALP activity and calcified nodules (Fig. 2E and F). To further verify the potential roles of MALAT1 in osteoporosis, we further determined the effects of MALAT1 on the cellular function of MC3T3-E1 cells. As shown in Fig. 2G, the increase in the apoptosis of MC3T3-E1 cells induced by MG was alleviated by overexpressed MALAT1. Moreover, upregulation of MALAT1 increased the protein expression of pro-proliferation gene, Bcl-2 and decreased apoptosis-related genes such as Bax and Caspase-3 (Fig. 2H).

\section{Overexpression of MALAT1 alleviated syndromes of osteoporosis}

To further clarify the roles of MALAT1 in osteoporosis, we detected the effects of MALAT1 overexpression on osteoporosis in vivo. The results from Micro-CT assays showed that the bone mineral density was 
decreased in HU group; however, overexpressed MALAT1 increased the bone mineral density compared with $\mathrm{HU}+$ vector group. Moreover, the decrease of the ratio of bone volume to total volume (BV/TV), trabecular bone number (Tb.N) and trabecular thickness (Tb.Th), and the increase in the values of the bone trabecula separation (Tb. Sp) and trabecular bone pattern factor (Tb.PF) induced by HU was alleviated by overexpressed MALAT1.

\section{MALAT1 served as a sponge to miR-485-5p}

LncRNAs functions as a ceRNAs via sponging miRNAs. Figure 4A showed the binding sites between MALAT1 and miR-485-5p. Moreover, miR-485-5p mimic significantly decreased the luciferase activity in the WT-MALAT1 group compared with mimic nc, but there was no significant difference in the MUTMALAT1 group (Fig. 4B). RNA pull-down further confirmed the interaction between MALAT1 and miR-485$5 p$ (Fig. 4C). The expression of miR-485-5p was significantly upregulated by silenced MALAT1 and downregulated by overexpressed MALAT1 (Fig. 4D). Moreover, miR-485-5p expression was elevated and was in negatively correlation with MALAT1 in HU mice (Fig. 4E and F). Additionally, the expression of miR485-5p was increased by MG in a time-dependent manner (Fig. 4G).

\section{MALAT1 promoted the osteogenic differentiation via sponging miR-485-5p}

As shown in Fig. 5A-C, overexpression of miR-485-5p decreased the expression of ALP, Bglap, Runx2, and Col1a1. As illustrated in Fig. 5B-E, the expression of Bmp4, Col1a1, and Spp1 at both mRNA and protein levels were elevated by MALAT1, which were rescued by miR-485-5p. Moreover, the increase in ALP activity induced by MALAT1 was abolished by miR-485-5p mimic (Fig. 5D). This was in consistent with the results from Alizarin red staining. As shown in Fig. 5E, overexpressed miR-485-5p significantly decreased calcium deposit of MC3T3-E1 cells (Fig. 5E). Moreover, miR-485-5p significantly increased the apoptosis rates of MC3T3-E1 cells as well as increased the expression of apoptosis-related genes, such as Bax and Caspase-3 and decreased Bcl-2 (Fig. 5F and G).

\section{WNT7B was a target of miR-485-5p}

To further explore the mechanism of miR-485-5p, Starbase (http://starbase.sysu.edu.cn/index.php) and TargetScan were applied to predict the target of miR-485-5p (Fig. 6A). Figure 6B showed the binding sites between miR-485-5p and WNT7B. The interaction between miR-485-5p and WNT7B was further verified by luciferase and RNA pull-down assay (Fig. 6C and D). GO analysis showed that WNT7B was a regulator of cellular signal transmission, cell growth and metabolisms (Fig. 6E). Inhibition of miR-485-5p significantly upregulated the expression of WNT7B, while overexpression of miR-485-5p downregulated WNT7B level (Fig. 6F). The expression of WNT7B was lower in HU mice than in the NC group (Fig. 6G). Moreover, WNT7B expression was positively correlated with MALAT1 (Fig. 6H). WNT7B expression was downregulated by MG in a time-dependent manner (Fig. 6I).

Inhibition of miR-485-5p promoted osteogenic differentiation by targeting WNT7B. 
Downregulated WNT7B alleviated the effects of miR-485-5p inhibitor on the expression of ALP, Bglap, Runx2, and Col1a1 (Fig. 7A-C). Moreover, ALP staining and Alizarin red staining showed that WNT7B knockdown significantly suppressed ALP activity and calcium deposit of MC3T3-E1 cells (Fig. 7D and E). Additionally, WNT7B knockdown suppressed the apoptosis of MC3T3-E1 cells as well as alleviated the effects on the protein expression of Bcl-2, Bax, Caspase-3 (Fig. 7F and G).

\section{Discussion}

These findings verified the protective roles of MALAT1 in osteoporosis. MALAT1 was downregulated in osteoporosis models in vivo and in vitro. Its downregulation suppressed the osteogenesis and promoted the apoptosis of MC3T3-E1 cells. This further exacerbated the development of osteoporosis, which was characterized by the decrease of MC3T3-E1 cells. However, overexpresion of MALAT1 promoted osteogenesis. This may thanks to its ceRNA properties. MALAT1 sponged miR-485-5p to activate WNT7B. These results further identified that MALAT1 can be a therapeutic target for osteoporosis.

The bone-protective roles of MALAT1 seem surprising. Previous studies report that MALAT1 functions as an oncogene in orthopedic malignancies and exerts pro-inflammatory properties in bone disorders, including osteoarthritis, osteolysis and ossification (12, 13, 20-22). However, emerging evidence reveal that MALAT1 protects against osteonecrosis of the femoral head and osteoporosis. This may due to its positive roles in osteogenesis. For instance, mesenchymal stem cell derived MALAT1 promotes human osteogenic differentiation and suppresses the development of osteoporosis. MALAT1 promotes osteoblast differentiation of adipose-derived mesenchymal stem cells, which further induces bone tissue repair and regeneration. The present study further verified the beneficial roles in treating osteoporosis. MALAT1 was downregulated in osteoporosis, and its downregulation was associated with the degradation of MC3T3-E1 cells. However, Upregulated MALAT1 increased the expression of osteogenesis related genes, such as ALP, RUNX2, and Col1a1, increased ALP activity and calcium deposition, and suppressed the apoptosis of MC3T3-E1 cells. These results suggested that MALAT1 exerted its protective roles in osteoporosis via promoting osteogenic differentiation of MC3T3-E1 cells. Stimulation of osteoblast and osteogenesis is pronounced in bone anabolic action (23), which plays a key role in developing bones. Osteogenesis imperfecta is deeply associated with the primary osteoporosis (24). Thence, osteogenesis may be the Achilles' heel of osteoporosis.

IncRNAs function as ceRNA to regulate gene expression and biological processes via sponging micrRNAs. Dysregulated IncRNAs modulate osteogenic differentiation, proliferation, apoptosis, adipogenesis, bone formation, necrosis. In this study, dysregulated MALAT1 contributed to the degradation of MC3T3-E1 cells and the development of osteoporosis via sponging miR-485-5p. miR-485$5 p$ was upregulated in osteoporosis patients (25). However, its downregulation promoted the osteogenic differentiation of human bone marrow-derived mesenchymal stem cells (26). Therefore, miR-485-5p exacerbate the development of osteoporosis. In this study, overexpression of miR-485-5p supressed the osteogenic differentiation and suppressed the apoptosis of MC3T3-E1 cells. These results suggested that MALAT1 alleviated osteoporosis by sponging miR-485-5p. 
WNT7B, a member of WNT family, facilitates bone formation. Chen et al. (18) reveal that WNT7B promotes glucose consumption and osteoblast differentiation. WNT7B induces the mineralization of the subsequent bone callus and trabecular and endosteal bone formation. Moreover, WNT7B promotes selfrenewal and osteogenic differentiation of bone marrow mesenchymal stem cells (27). These results dictates that WNT7B may play a beneficial role in osteoporosis via regulating metabolism processes. This bone anabolic function may be a promising therapy for osteoporosis. In this study, MALAT1 sponged miR-485-5p to activate WNT7B, which was downregulated in osteoporosis model in vitro. WNT7B knockdown suppressed osteogenic differentiation of bone marrow mesenchymal stem cells. These results further verified that WNT7B protected against osteoporosis, which is consistent with previous studies. WNT7B modulates glucose metabolism via upregulating Glut1 (19). Chen et al (27) demonstrate that WNT7B exerts its bone anabolic function via activating Sox11. Therefore, we further investigated the underlying mechanisms. Therefore, further studies are needed to unveil the underlying molecular mechanisms.

\section{Conclusions}

Low expression of MALAT1 was closely associated with the development of osteoporosis. Overexpression of MALAT1 promoted osteogenic differentiation of bone marrow mesenchymal stem cells via avtivating miR-485-5p/WNT7B axis. This may be a promising therapy for osteoporosis.

\section{Abbreviations}

$\mathrm{HU}$

hindlimb unloading

MG

Microgravity

MALAT1

metastasis-associated lung adenocarcinoma transcript 1

WNT7B

Wnt family member 7B

$\mathrm{BV} / \mathrm{TV}$

the ratio of bone volume to total volume (BV/TV)

Tb.N

trabecular bone number

Tb.Th

trabecular thickness (Tb.Th)

Tb. Sp

bone trabecula separation

Tb.PF

trabecular bone pattern factor 


\section{Declarations}

\section{Ethics approval and consent to participate}

This study was approved by the Ethics Committee of China-Japan Union Hospital of Jilin University.

Consent for publication

Not applicable.

\section{Availability of data and materials}

The datasets used and/or analysed during the current study are available from the corresponding author on reasonable request.

\section{Competing interests}

The authors declare that they have no competing interests.

\section{Funding}

Not applicable.

\section{Authors' contributions}

Yuan Zhou have drafted the work and substantively revised it. Zhuo Xu and Yuanyi Wang were responsible for the acquisition and analysis of data, and worked with Yuan Zhou and Qiang Song to interpret the data. Ruofeng Yin provided the conception and design of the work. All authors read and approved the final manuscript.

\section{Acknowledgements}

Not applicable.

\section{Authors' information}

Not applicable.

\section{References}

1. Lane JM, Russell L, Khan SN. Osteoporosis. Clin Orthop Relat Res 2000 Mar;(372):139-50. doi:10.1097/00003086-200003000-00016.

2. Wang Y, Tao Y, Hyman ME, Li J, Chen Y. Osteoporosis in china. Osteoporos Int. 2009 Oct;20(10):1651-62. doi:10.1007/s00198-009-0925-y. 
3. Srivastava M, Deal C. Osteoporosis in elderly: prevention and treatment. Clin Geriatr Med. 2002 Aug;18(3):529-55. doi:10.1016/s0749-0690(02)00022-8.

4. Lee WC, Guntur AR, Long F, Rosen CJ. Energy Metabolism of the Osteoblast: Implications for Osteoporosis. Endocr Rev. 2017 Jun 1;38(3):255-266. doi: 10.1210/er.2017-00064.

5. Chen X, Wang Z, Duan N, Zhu G, Schwarz EM, Xie C. Osteoblast-osteoclast interactions. Connect Tissue Res. 2018 Mar;59(2):99-107. doi: 10.1080/03008207.2017.1290085.

6. Lynda F, Bonewald. The amazing osteocyte. J Bone Miner Res. 2011 Feb;26(2):229-38. doi:10.1002/jbmr.320.

7. Ulitsky I, Bartel DP. lincRNAs: genomics, evolution, and mechanisms. Cell. 2013 Jul 3;154(1):26-46. doi: 10.1016/j.cell.2013.06.020.

8. Yang Y, Yujiao W, Fang W, Linhui Y, Ziqi G, Zhichen W, Zirui W, Shengwang W. The roles of miRNA, IncRNA and circRNA in the development of osteoporosis. Biol Res. 2020 Sep 16;53(1):40. doi: 10.1186/s40659-020-00309-z.

9. He T, Liu W, Cao L, Liu Y, Zou Z, Zhong Y, Wang H, Mo Y, Peng S, Shuai C. CircRNAs and LncRNAs in Osteoporosis. Differentiation. 2020 Nov-Dec;116:16-25. doi: 10.1016/j.diff.2020.10.002.

10. Patil S, Dang K, Zhao X, Gao Y, Qian A. Role of LncRNAs and CircRNAs in Bone Metabolism and Osteoporosis. Front Genet. 2020 Nov 13;11:584118. doi: 10.3389/fgene.2020.584118.

11. Yang X, Yang J, Lei P, Wen T. LncRNA MALAT1 shuttled by bone marrow-derived mesenchymal stem cells-secreted exosomes alleviates osteoporosis through mediating microRNA-34c/SATB2 axis. Aging (Albany NY). 2019 Oct 26;11(20):8777-8791. doi: 10.18632/aging.102264.

12. Zheng $S$, Wang YB, Yang YL, Chen BP, Wang CX, Li RH, Huang D. LncRNA MALAT1 inhibits osteogenic differentiation of mesenchymal stem cells in osteoporosis rats through MAPK signaling pathway. Eur Rev Med Pharmacol Sci. 2019 Jun;23(11):4609-17. doi:10.26355/eurrev_201906_18038.

13. Gao Y, Xiao F, Wang C, Wang C, Cui P, Zhang X, Chen X. Long noncoding RNA MALAT1 promotes osterix expression to regulate osteogenic differentiation by targeting miRNA-143 in human bone marrow-derived mesenchymal stem cells. J Cell Biochem. 2018 Aug;119(8):6986-6996. doi: $10.1002 / j c b .26907$.

14. Aguilera KY, Dawson DW. WNT Ligand Dependencies in Pancreatic Cancer. Front Cell Dev Biol. 2021;9:671022.

15. Tang C, Wang X, Ji C, Zheng W, Yu Y, Deng X, Zhou X, Fang L. The Role of miR-640: A Potential Suppressor in Breast Cancer via Wnt7b/ $\beta$-catenin Signaling Pathway. Front Oncol. 2021;11:645682.

16. Song D, He G, Song F, Wang Z, Liu X, Liao L, Ni J, Silva MJ, Long F. Inducible expression of Wnt7b promotes bone formation in aged mice and enhances fracture healing. Bone Res. 2020;8:4.

17. Matsuoka K, Bakiri L, Wolff LI, Linder M, Mikels-Vigdal A, Patiño-García A, Lecanda F, Hartmann C, Sibilia M, Wagner EF. Wnt signaling and Loxl2 promote aggressive osteosarcoma. Cell Res. 2020;30(10):885-901. 
18. Chen H, Song F, Long F. WNT7B overexpression rescues bone loss caused by glucocorticoids in mice. FASEB J. 2021;35:e21683.

19. Chen H, Ji X, Lee WC, Shi Y, Li B, Abel ED, Jiang D, Huang W, Long F. Increased glycolysis mediates Wnt7b-induced bone formation. FASEB J. 2019;33:7810-21.

20. Liu C, Ren S, Zhao S, Wang Y. LncRNA MALAT1/MiR-145 Adjusts IL-1 $\beta$-Induced Chondrocytes Viability and Cartilage Matrix Degradation by Regulating ADAMTS5 in Human Osteoarthritis. Yonsei Med J. 2019 Nov;60(11):1081-92.

21. Yang $X$, Zhang $Y, L i Y$, Wen T. MALAT1 enhanced the proliferation of human osteoblasts treated with ultra-high molecular weight polyethylene by targeting VEGF via miR-22-5p. Int J Mol Med. 2018 Mar;41(3):1536-46.

22. Yuan X, Guo Y, Chen D, Luo Y, Chen D, Miao J, Chen Y. Long non-coding RNA MALAT1 functions as miR-1 sponge to regulate Connexin 43-mediated ossification of the posterior longitudinal ligament. Bone. 2019 Oct;127:305-14.

23. Estell EG, Rosen CJ. Emerging insights into the comparative effectiveness of anabolic therapies for osteoporosis. Nat Rev Endocrinol. 2021;17(1):31-46.

24. El-Gazzar A, Högler W. Mechanisms of Bone Fragility: From Osteogenesis Imperfecta to Secondary Osteoporosis. Int J Mol Sci. 2021 Jan 10;22(2):625.).

25. Asila A, Yang X, Kaisaer Y, Ma L. SNHG16/miR-485-5p/BMP7 axis modulates osteogenic differentiation of human bone marrow-derived mesenchymal stem cells. J Gene Med. 2021 Mar;23(3):e3296.

26. Zhang SY, Gao F, Peng CG, Zheng CJ, Wu MF. miR-485-5p promotes osteoporosis via targeting Osterix. Eur Rev Med Pharmacol Sci. 2018 Aug;22(15):4792-9.

27. Yu F, Wu F, Li F, Liao X, Wang Y, Li X, Wang C, Shi Y, Ye L. Wnt7b-induced Sox11 functions enhance self-renewal and osteogenic commitment of bone marrow mesenchymal stem cells. Stem Cells. 2020 Aug;38(8):1020-33.

\section{Figures}



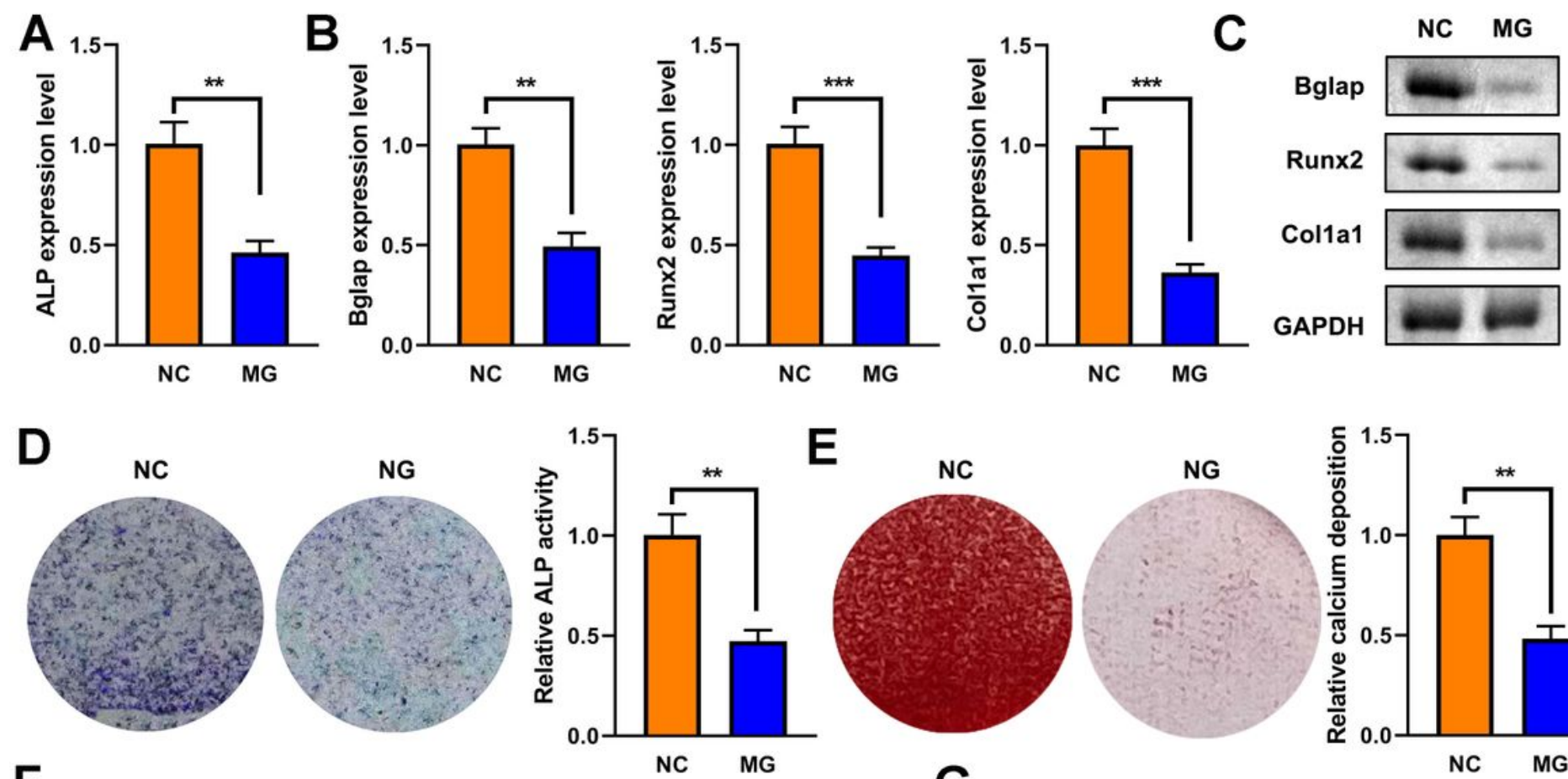

$\mathbf{F}$
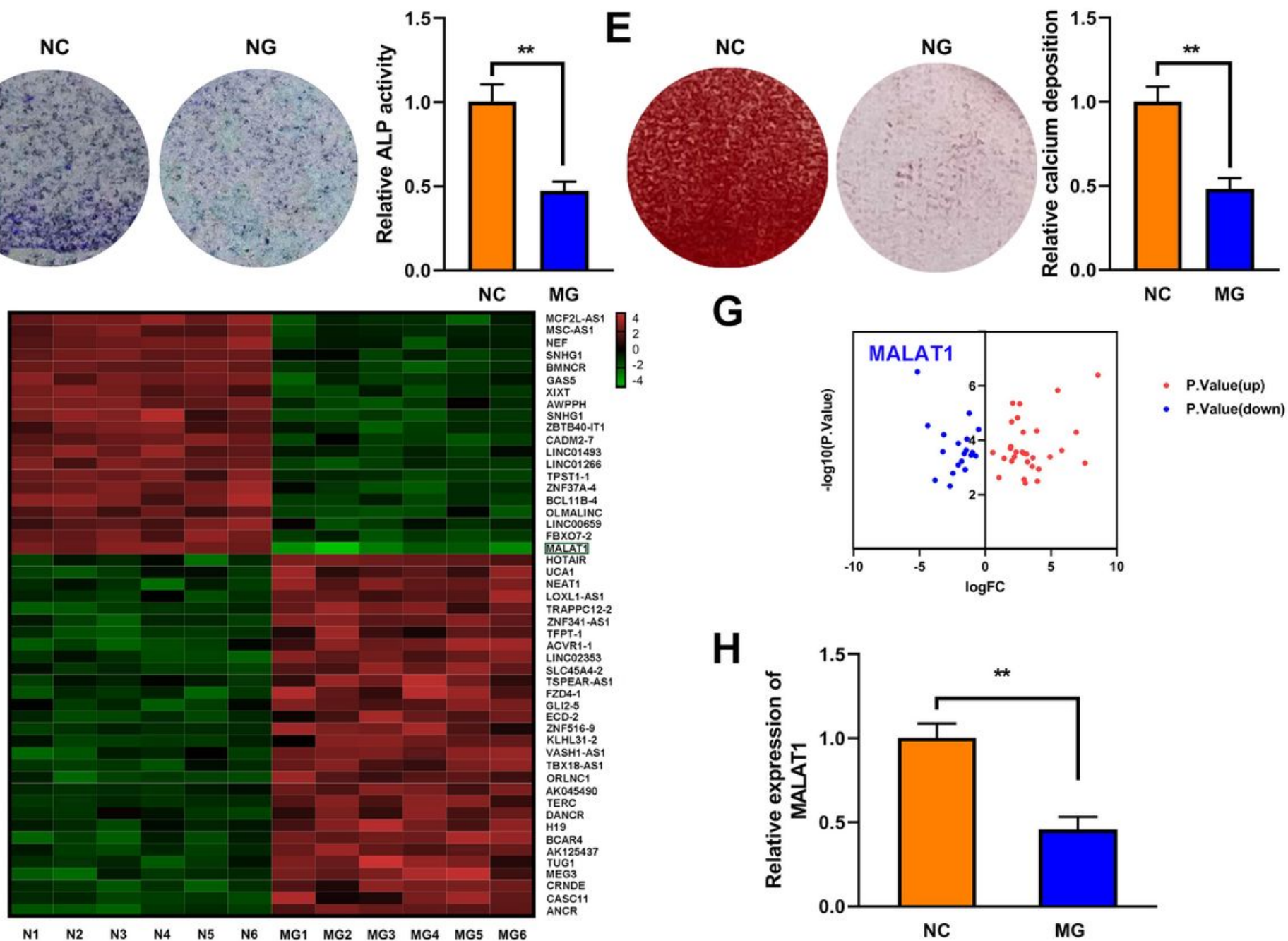

\section{Figure 1}

The expression of MALAT1 was downregulated MG-treated cells. (A) The expression of ALP determined using RT-qPCR. (B) The mRNA expression of Bglap, Runx2, and Col1a1 were measured by RT-qPCR. (C) The protein expression of Bglap, Runx2, and Col1a1 was detected using western blot. (D) ALP activity was detected using ALP staining. (E) Alizarin red staining was used to measure calcium deposit. ( $F$ and G) The differentially expressed IncRNAs in MG-treated MC3T3-E1 cells. $(\mathrm{H})$ The expression of MALAT1 in MG-treated MC3T3-E1 cells was detected using RT-qPCR. ${ }^{\star *} P<0.01$, ${ }^{\star \star *} P<0.001$. 

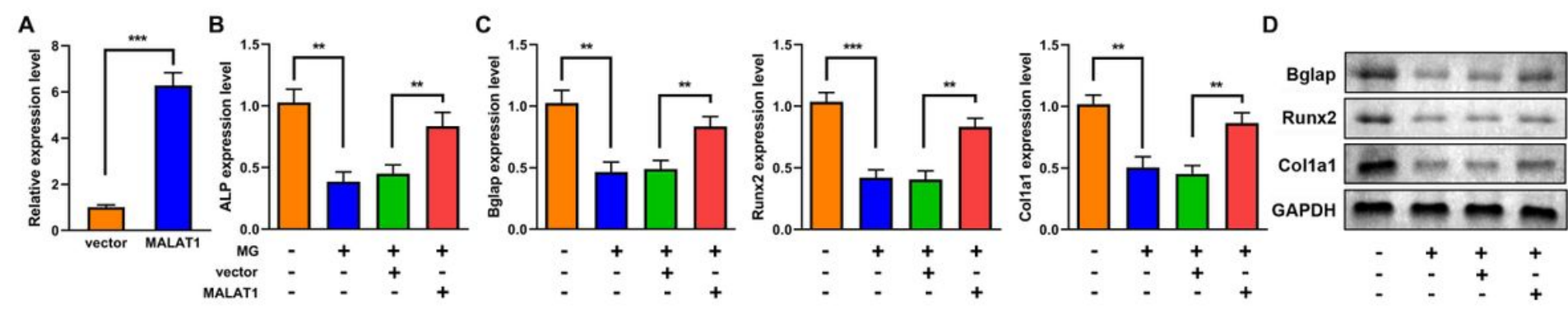

$\mathbf{E}$
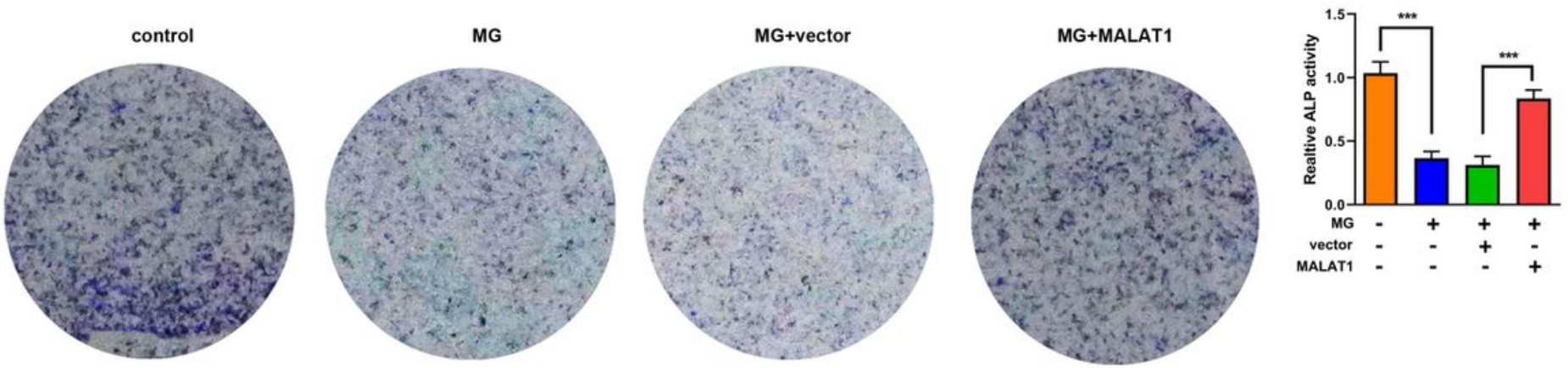

$\mathbf{F}$
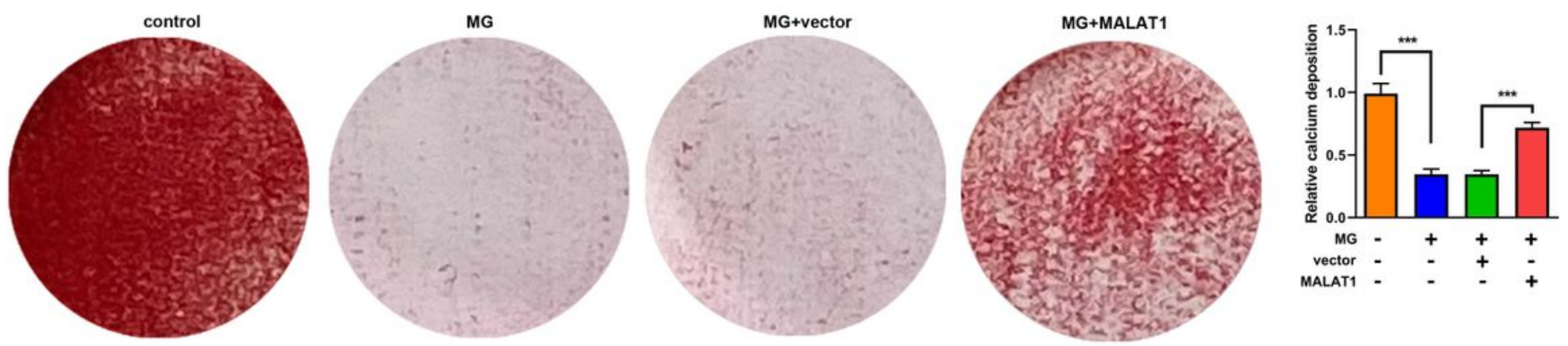

G

H

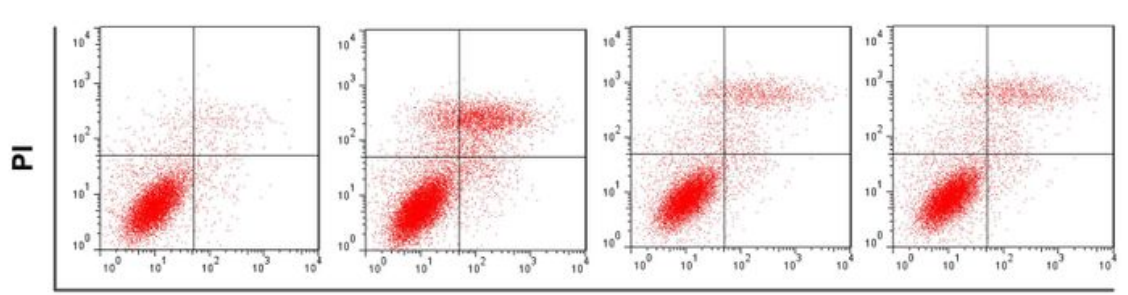

Annexin V-FITC

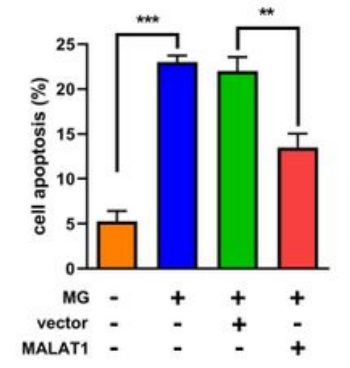

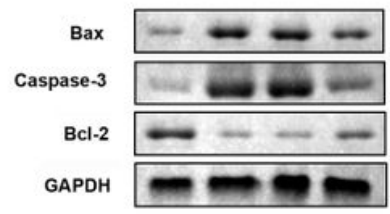

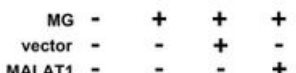

Figure 2

MALAT1 facilitated MC3T3-E1 cell osteogenic differentiation and suppressed apoptosis. (A) The expression of MALAT1 was determined using RT-qPCR. (B) The transfection efficiency of MALAT1. (C) The expression of ALP. (D) The mRNA expression of Bglap, Runx2, and Col1a1. (E) The protein expression of Bglap, Runx2, and Col1a1. (F) The ALP activity of MC3T3-E1 cells. (G) Alizarin red staining was used to measure calcium deposit. (I) Cell apoptosis was analyzed by flow cytometry, and cell apoptosis rate was quantified. $(\mathrm{H})$ The protein expression of Bax, caspase-3, and Bcl-2. ${ }^{*} \mathrm{P}<0.05,{ }^{\star} \mathrm{P}<0.01,{ }^{\star} * \star \mathrm{P}<0.001$. 
A

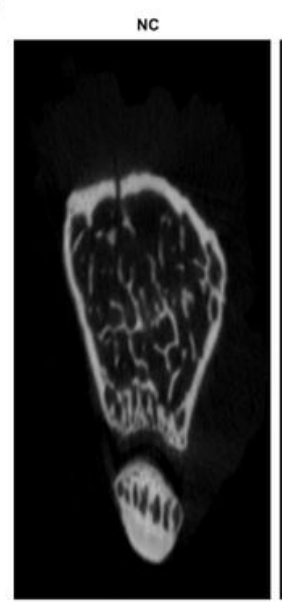

HU

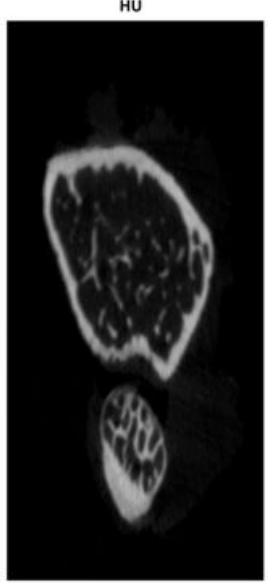

HU+vector

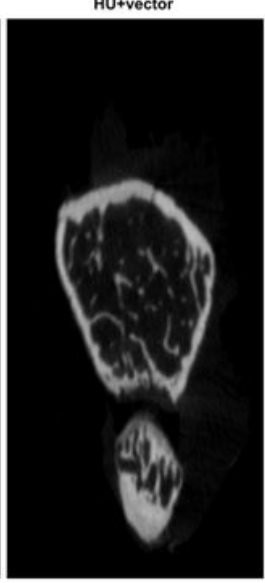

B

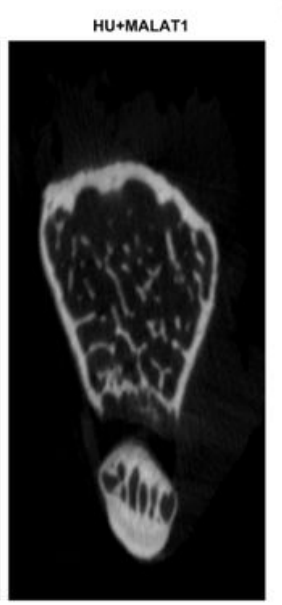

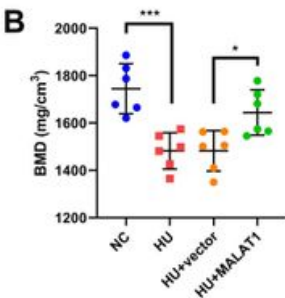
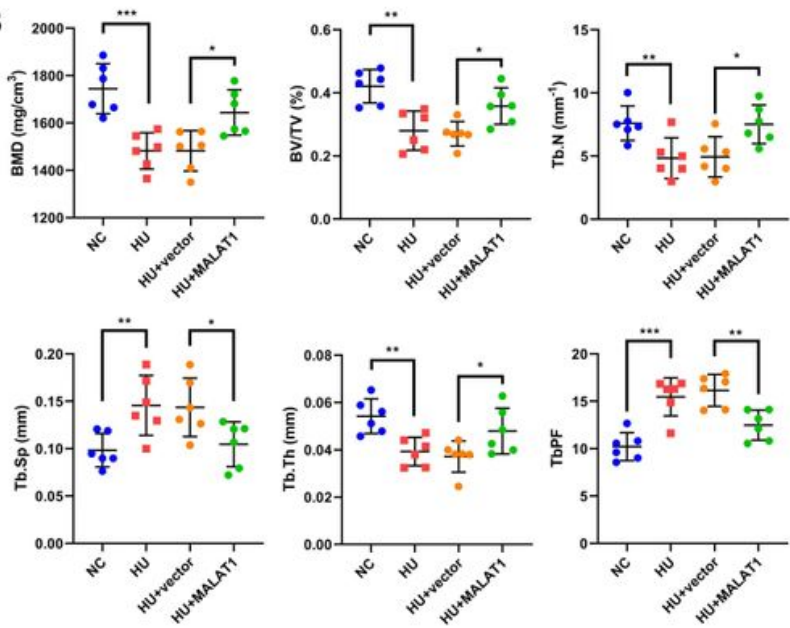

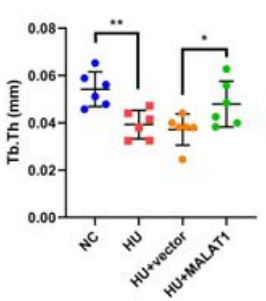

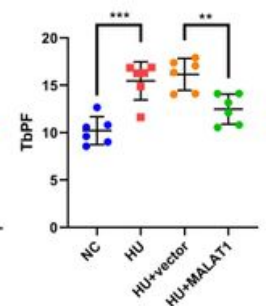

Figure 3

MALAT1 improved bone phenotype in HU mice. (A) Micro-CT images of distal femurs of mice. The (B) BMD, BV/TV, Tb.N, Tb.Sp, Tb.Th, and TbPF were analyzed by Micro-CT. ${ }^{*} P<0.05,{ }^{*}{ }^{*}<<0.01,{ }^{*} * * P<0.001$.

\begin{abstract}
A
IncMALAT1 wt: 5' GUCAAGAGAAGUGUCAGCCUCA 3' miR-485-5p: 3' CUUAAGUAGUGCCGGUCGGAGA 5' IncMALAT1 mut: 5' GUCAAGAGAAGUGUCACGGAGA 3'
\end{abstract}

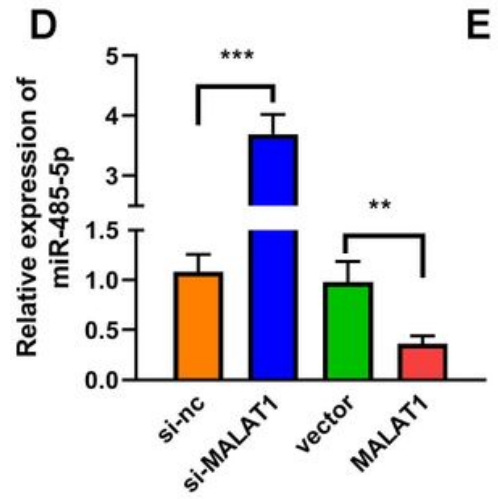

\section{$B$
$3^{\prime}$
$3^{\prime}$}

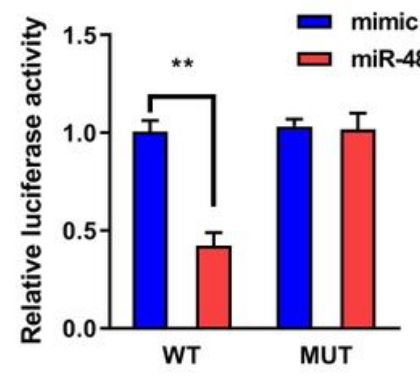

E

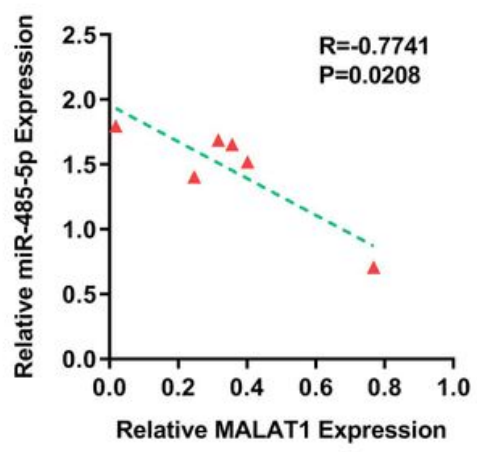

$\mathbf{F}$

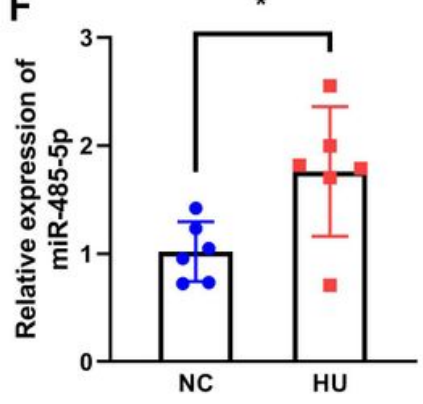

C

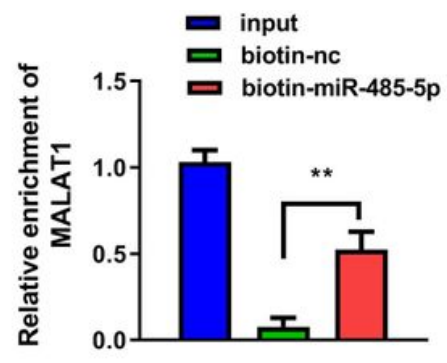

G

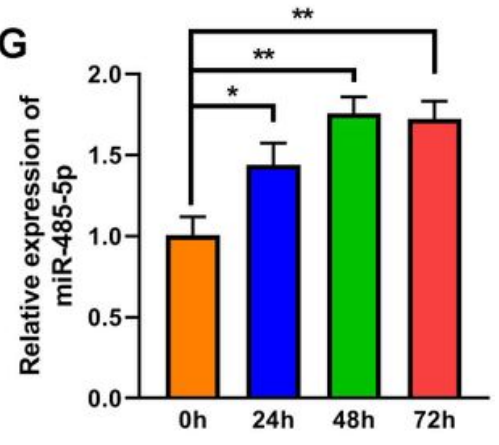

Figure 4

MALAT1 acted as a miRNA sponge of miR-485-5p. (A) The binding sites of wild-type (wt) MALAT1 and miR-485-5p were shown. Meanwhile, mutant (mut) MALAT1 sequences were also shown. (B) The targeting relationship was confirmed by luciferase reporter assay. (C) The targeted relationship was verified using RNA pull-down. (D) The expression of miR-485-5p was measured by RT-qPCR after overexpression or knockdown of MALAT1. (E) The expression of miR-485-5p was detected using RTqPCR. (F) Pearson method was applied for correlation analysis. (G) The expression of miR-485-5p was detected using RT-qPCR. ${ }^{*} P<0.05,{ }^{*} \mathrm{P}<0.01$, ${ }^{* *} \mathrm{P}<0.001$. 

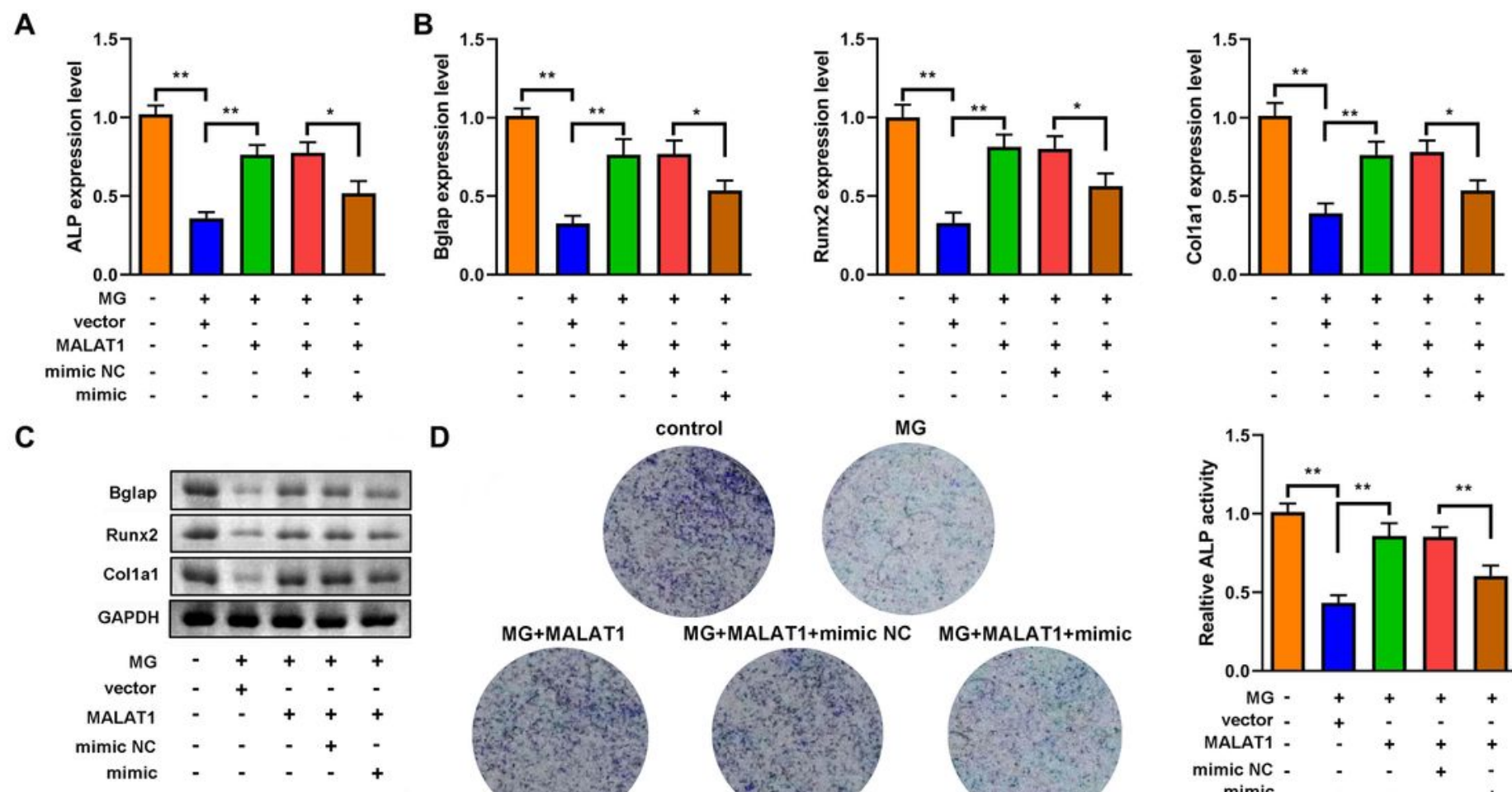

D
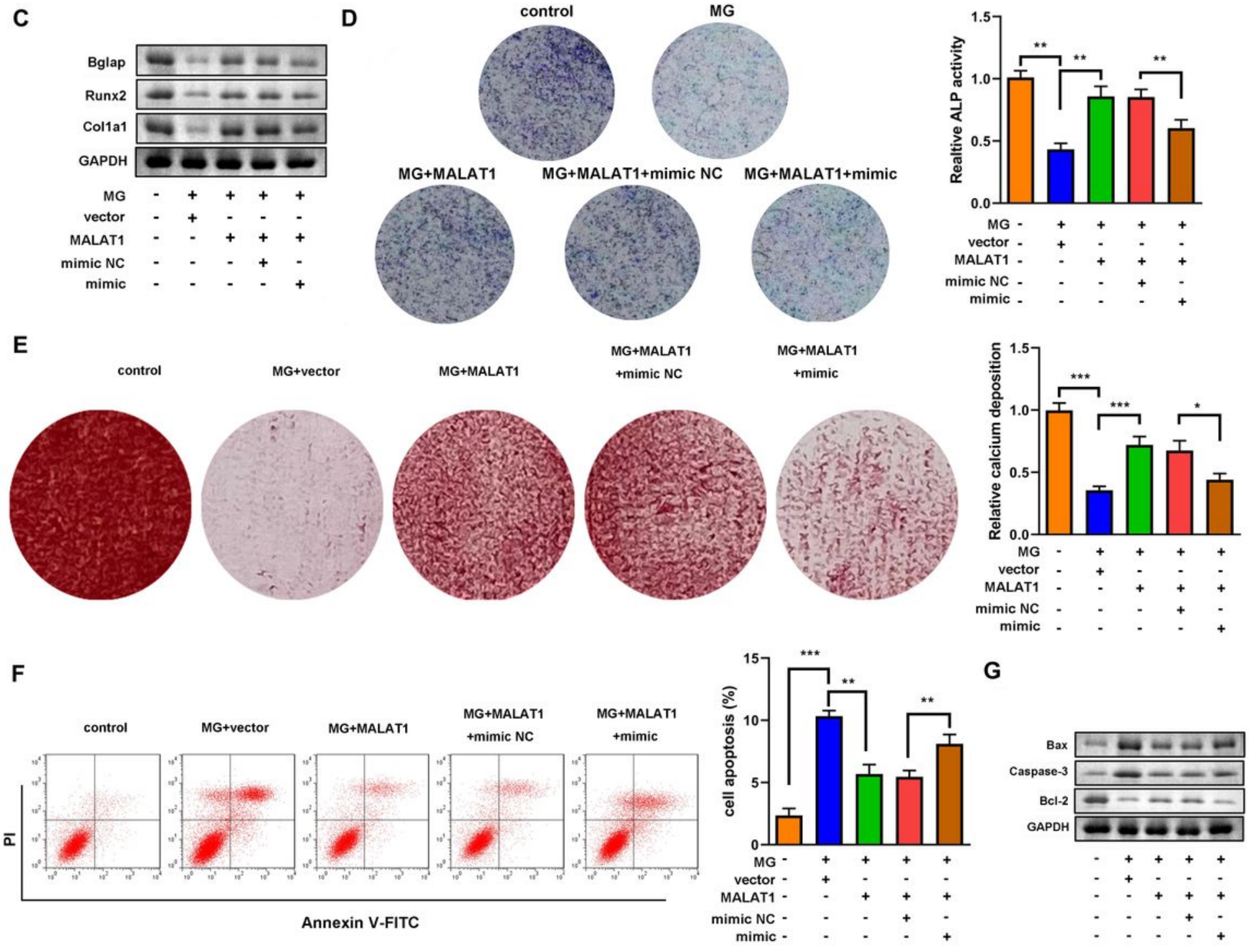

G

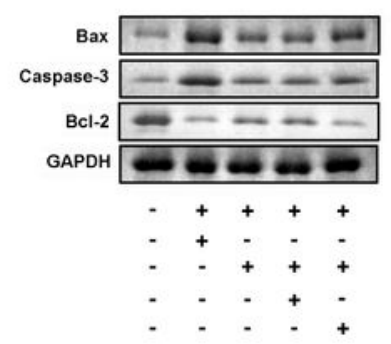

Figure 5

miR-485-5p attenuated osteogenic differentiation induced by MALAT1. (A) The expression of ALP. (B) The expression of Bglap, Runx2, and Col1a1 was detected by RT-qPCR. (C) The protein expression of Bglap, Runx2, and Col1a1. (D) The ALP activity of MC3T3-E1 cells. (E) The calcium deposit detected using Alizarin red staining. (F) The apoptosis of MC3T3-E1 cells detected using flow cytometry. (G) The protein expression of Bax, Caspase-3, and Bcl-2 detected using western blot. ${ }^{*} \mathrm{P}<0.05,{ }^{*} \mathrm{P}<0.01,{ }^{*} * \mathrm{P}<0.001$. 
A

Targetscann 7.2

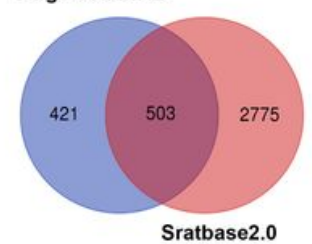

E

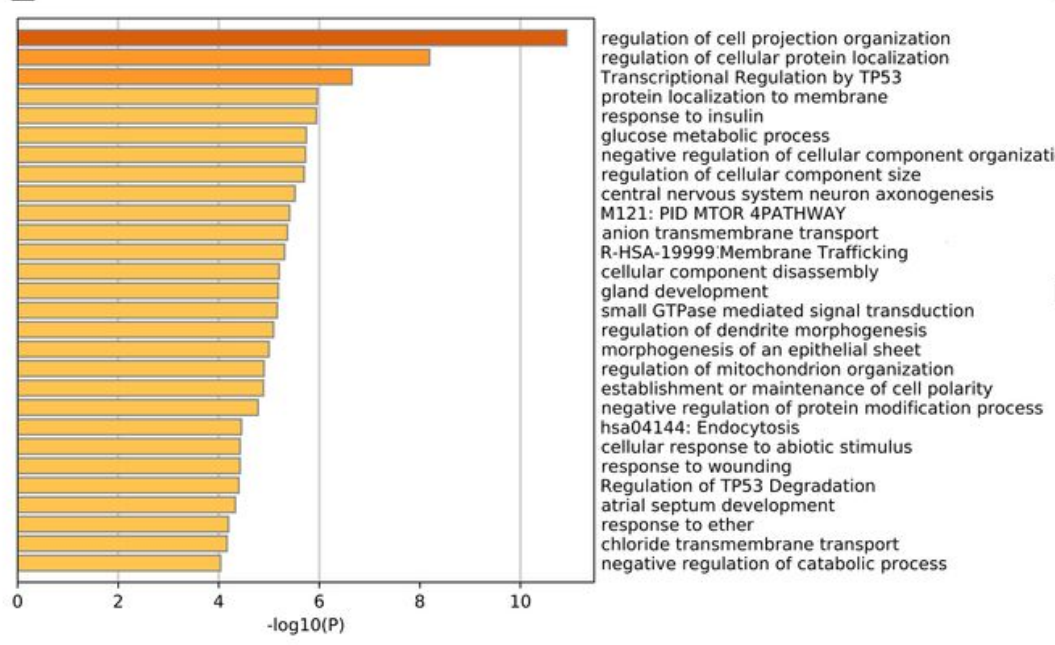

WT- WNT7B: 5' aGUCCAUAGGCCCGG-CAGCCUCU 3'

| || | | | | | |

miR-485-5p: 3' cuUAAGUA----GUGCCGGUCGGAGA 5'

MUT-WNT7B: 5' aGUCCAUAGGCCCGG-CUCGGAGU 3'
C

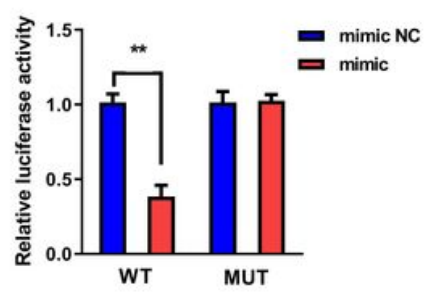

$\mathbf{F}$

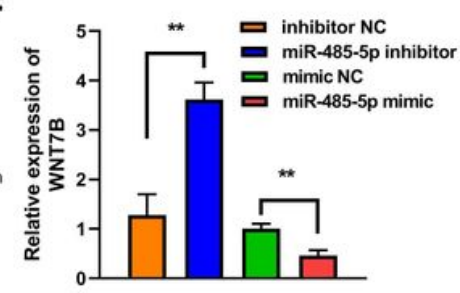

$\mathbf{H}$

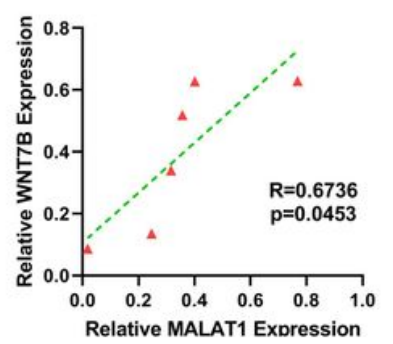

D

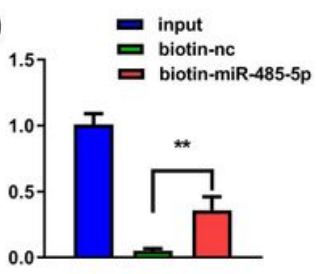

G
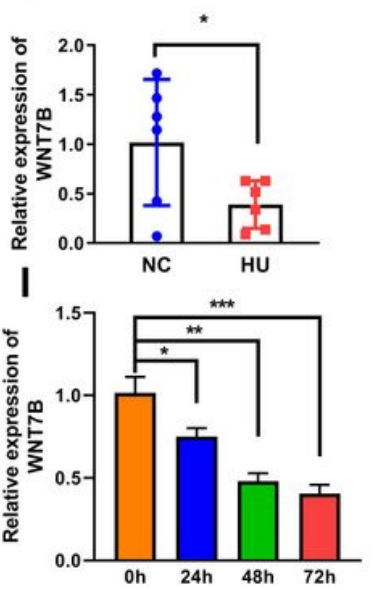

Figure 6

WNT7B is a direct target of miR-485-5p. (A) The potential target predicted by Starbase2.0 and TargetScan7.2. (B) Potential binding sites for miR-485-5p to WNT7B were predicted by TargetScan. (C) The targeted relationship was confirmed by dual-luciferase reporter assay. (D) The targeted relationship was confirmed by RNA pull-down. (E) GO analysis of biological processes. (F) The expression of WNT7B was assessed by RT-qPCR. (G) The expression of WNT7B in vivo. (H) The correlation between the expression of WNT7B and MALAT1 analyzed using Pearson analysis. (I) The expression of WNT7B detected using RT-qPCR. ${ }^{*}<<0.05 .{ }^{\star}{ }^{*} \mathrm{P}<0.01$. 

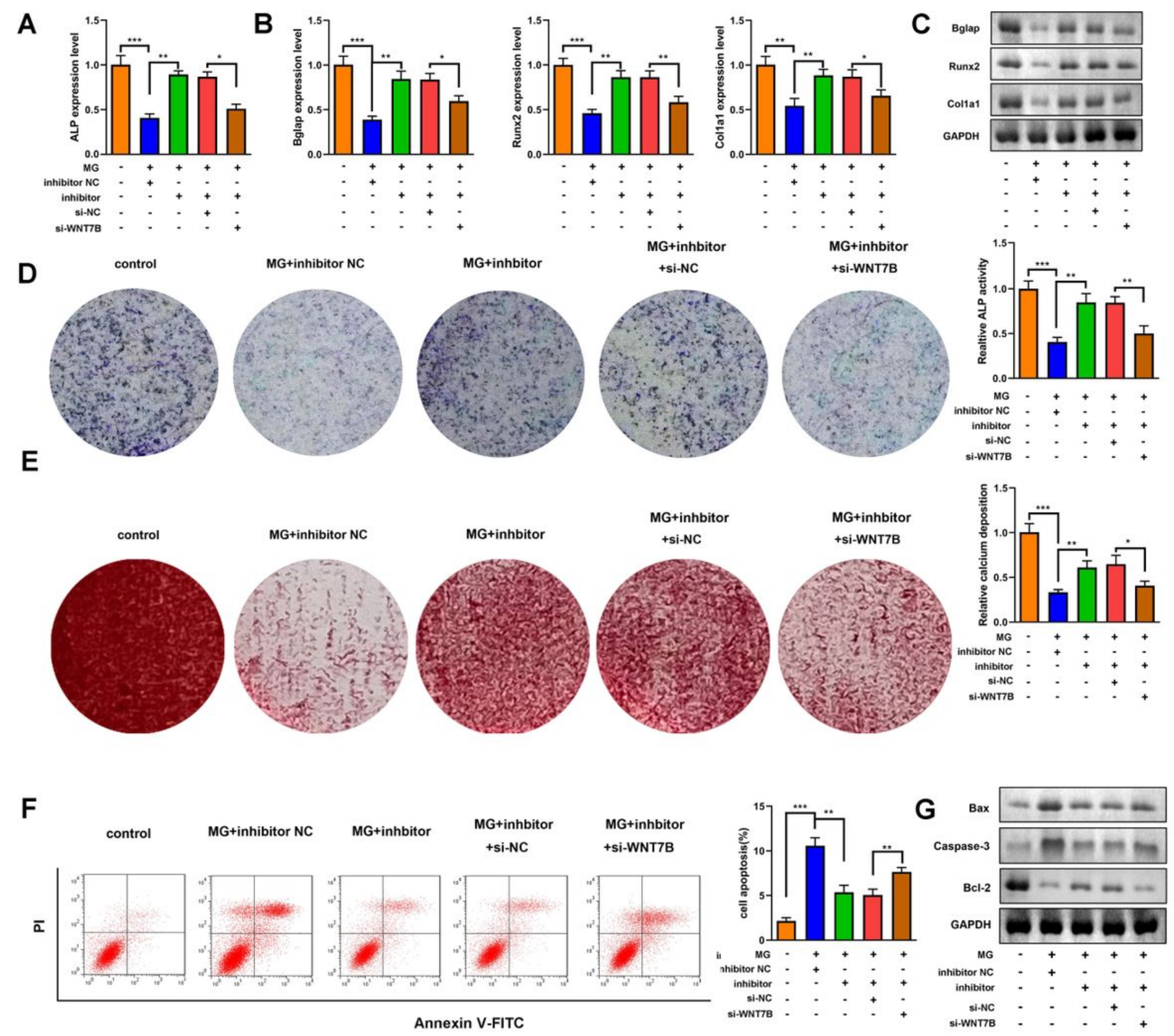

\section{Figure 7}

WNT7B was a negative regulator of miR-485-5p on the osteogenic differentiation. (A) The expression of ALP. (B) The expression of Bglap, Runx2, and Col1a1 was detected by RT-qPCR. (C) The protein expression of Bglap, Runx2, and Col1a1. (D) The ALP activity of MC3T3-E1 cells. (E) The calcium deposit detected using Alizarin red staining. (F) The apoptosis of MC3T3-E1 cells detected using flow cytometry. (G) The protein expression of Bax, Caspase-3, and $\mathrm{Bcl}-2$ detected using western blot. ${ }^{*} \mathrm{P}<0.05,{ }^{\star \star} \mathrm{P}<0.01$, $\star * * P<0.001$. 